\title{
Growth hormone level at admission and its evolution during refeeding are predictive of short-term outcome in restrictive anorexia nervosa
}

\author{
Juan P. Nogueira ${ }^{1}$, René Valéro ${ }^{1,2 *}$, Marie Maraninchi ${ }^{1}$, Anne M. Lorec ${ }^{3}$, Catherine Samuelian-Massat ${ }^{2}$, \\ Audrey Bégu-Le Corroller ${ }^{2}$, Alain Nicolay ${ }^{1,3}$, Jean Gaudart ${ }^{4}$, Henri Portugal ${ }^{1,3}$ and Bernard Vialettes ${ }^{1,2}$ \\ ${ }^{1}$ UMR 1062 INSERM/1260 INRA, Aix-Marseille University, Marseille, France \\ ${ }^{2}$ Department of Nutrition, Metabolic Diseases and Endocrinology, APHM, La Timone Hospital, Aix-Marseille University, \\ 264, Rue Saint-Pierre, 13005 Marseille, France \\ ${ }^{3}$ Department of Biochemistry, APHM, La Timone Hospital, Aix-Marseille University, Marseille, France \\ ${ }^{4}$ Biostatistics Research Unit (LERTIM), Faculty of Medicine, APHM, Aix-Marseille University, Marseille, France
}

(Submitted 1 February 2012 - Final revision received 17 August 2012 - Accepted 17 August 2012 - First published online 2 November 2012)

\section{Abstract}

The growth hormone (GH)-insulin-like growth factor-1 (IGF-1) axis is dramatically altered in patients with anorexia nervosa (AN). The aim of the present study was to investigate whether GH and IGF-1 could be predictors of outcome in patients with a restrictive form of AN. Blood levels of GH, IGF-1, adipocytokines, ghrelin, insulin, glucose, and sex and thyroid hormones were measured in eleven women inpatients with AN and in ten healthy women controls. Three stages were compared during refeeding: admission (T0), when BMI reached $16 \mathrm{~kg} / \mathrm{m}^{2}$ (T1) and at discharge when BMI reached $17.5 \mathrm{~kg} / \mathrm{m}^{2}$ (T2). Clinical status was assessed 6 months after discharge from hospital (T3), and remission was defined by the maintenance of a BMI $\geq 17.5 \mathrm{~kg} / \mathrm{m}^{2}$. AN patients in remission (AN-R; $n$ 6) had significantly higher GH levels at admission than those who relapsed (AN-NR; $n 5)(P<0 \cdot 05)$. During refeeding $(\Delta=$ T2 - T0), the AN-R group differed from the AN-NR group only by both GH level decrease $(P<0 \cdot 05)$ and BMI increase $(P<0 \cdot 05)$. In multiple regression analysis, $\Delta \mathrm{GH}$ was associated negatively and significantly and $\Delta$ leptin and $\Delta$ body fat mass levels were associated positively and significantly with BMI at T3 and explained $88 \%$ of its variability $\left(r^{2} 0 \cdot 88, P<0.05\right)$. The present study suggests that a low GH level at admission and the absence of its decrease after weight recovery could predict short-term relapse in women suffering from a restrictive form of AN.

\section{Key words: Anorexia nervosa: Growth hormone: Insulin-like growth factor-1: Outcomes}

Anorexia nervosa (AN) is an eating disorder caused by voluntary restriction of energy intake, intensive physical exercise and/or purging behaviours, and is characterised by weight loss, mainly at the expense of adipose tissue ${ }^{(1,2)}$. These behaviours lead to multiple endocrine abnormalities commonly associated with starvation, including high ghrelin levels, hypogonadotropic hypogonadism and adipocytokine disturbances $^{(3,4)}$. Most of these anomalies are corrected with the recovery of normal nutritional conditions ${ }^{(5)}$.

Several studies have shown that the growth hormone (GH)-insulin-like growth factor-1 (IGF-1) axis was dramatically altered in patients with $\mathrm{AN}^{(6,7)}$. But, although Argente et $a l^{(6)}$ have discerned two different groups of anorexic patients, one group having hypersecretion of $\mathrm{GH}$ and one group with hyposecretion of $\mathrm{GH}$, they were unable to distinguish these two groups regarding either physical or psychological parameters at the time of diagnosis or during the recuperation period.

In the present study, the main objective was to investigate if GH and IGF-1 levels could represent prognostic factors of outcome in restrictive AN. To test this hypothesis, we performed a prospective study on a homogeneous group of eleven patients suffering from a severe pure restrictive form of AN and submitted to the same standardised inpatient protocol treatment. GH and IGF-1 levels, as well as hormone and adipocytokine profiles, insulin sensitivity, body composition and energy expenditure, were assessed at admission and at various stages of weight recuperation.

Abbreviations: AN, anorexia nervosa; AN-NR, anorexia nervosa-not in remission; AN-R, anorexia nervosa-remission; BFM, body fat mass; DSM-IV, Diagnostic and Statistical Manual of Mental Disorders, Fourth Edition; FGF-21, fibroblast growth factor-21; GH, growth hormone; HOMA-IR, homeostasis model assessment-insulin resistance; IGF-1, insulin-like growth factor-1; T0, admission (baseline); T1, BMI reached 16 kg/m²; T2, discharge when BMI reached $17.5 \mathrm{~kg} / \mathrm{m}^{2} ; \mathrm{T} 3$, assessment at 6 months after discharge.

*Corresponding author: R. Valéro, fax +33491386599, email rvalero@mail.ap-hm.fr 


\section{Materials and methods}

Subjects

The present study was conducted according to the guidelines laid down in the Declaration of Helsinki, and all procedures involving human patients were approved by the Research Ethics Board of Aix-Marseille University and all subjects gave verbal informed consent. Verbal consent was witnessed and formally recorded. A total of eleven women suffering from AN were recruited in our department of nutrition. All subjects met the Diagnostic and Statistical Manual of Mental Disorders, Fourth Edition (DSM-IV) criteria for the restrictive form of AN. This population was homogeneously selected as every patient included in this series suffered from the short-duration $(12 \cdot 2$ (SEM 5.0) weeks) and active (BMI loss rate: $-0 \cdot 2$ (SEM $0.03) \mathrm{kg} / \mathrm{m}^{2}$ per week) restrictive type of AN. Patients with relapsing AN or exhibiting binge eating-purging behaviours were excluded. The patients were referred to the inpatient unit due to the presence of at least one of the following conditions: life-threatening medical condition due to severe malnutrition, incapacity to reach weight objectives during ambulatory care or marked suicidal ideation. None of the patients used oral contraception for several weeks before the hospitalisation in our department. All patients were treated by a single team from the department of nutrition according to a structured inpatient protocol combining medical, nutritional and psychiatric supports based on widely accepted standards of practice ${ }^{(8,9)}$. Treatment was based on a weight contract (progressive return to near-normal BMI, i.e. $\geq 17.5 \mathrm{~kg} / \mathrm{m}^{2}$ ) and consisted of medical supervision, nutritional support by a dietitian, mixed cognitive behavioural and analytical psychotherapy sessions and art therapy, both performed twice a week. The standardised feeding protocol consisted of a progressive increment of energy intake from 3350 to $8370 \mathrm{~kJ} / \mathrm{d}$.

\section{Methods}

Patients were studied at three stages of care: at admission (T0), when BMI reached $16 \mathrm{~kg} / \mathrm{m}^{2}$ (T1), and at discharge, when BMI reached $17.5 \mathrm{~kg} / \mathrm{m}^{2}$ (T2). The diet of each patient was estimated by a dietitian at admission (T0) before refeeding. Clinical status was assessed 6 months after discharge from hospital (T3). Short-term remission was defined by the maintenance of a BMI $\geq 17.5 \mathrm{~kg} / \mathrm{m}^{2}$ at 6 months after discharge. A BMI $\geq 17.5 \mathrm{~kg} / \mathrm{m}^{2}$ was chosen to define remission because the diagnostic criteria for AN from the DSM-IV include a BMI $\leq 17.5 \mathrm{~kg} / \mathrm{m}^{2(10)}$. The weight achieved to reach a BMI $\geq 17.5 \mathrm{~kg} / \mathrm{m}^{2}$ at discharge was defined as the 'target weight' in the present study. In all, six patients exhibited remission (AN-R group) and five patients were characterised by a quick relapse, being unable to maintain their weight objective (AN-NR group). It was impossible to take into account 'the healthy weight' defined by the weight at the loss of menstruation because most of the patients (four of six patients in the remission group (AN-R group) and four of five in the not in remission group (AN-NR group)) were taking oral oestroprogestogenic treatment. In addition, ten normal-weight healthy women were recruited as controls after a medical interview and a physical examination by a physician specialised in nutrition. None of them had any history of eating disorders or other psychiatric illness.

Blood samples were obtained between 07.00 and 08.00 hours after an overnight fast. Plasma glucose was measured using the hexokinase oxidase method (Beckman Coulter ${ }^{\circledR}$ ) and plasma insulin levels were determined by electrochemiluminescence (Roche Diagnostics ${ }^{\circledR}$ ). Insulin resistance was estimated using homeostasis model assessment-insulin resistance $($ HOMA-IR $=$ fasting insulin $(\mathrm{mIU} / \mathrm{l}) \times$ fasting glucose $(\mathrm{mmol} / \mathrm{l}) /$ 22.5). Alanine aminotransferase and aspartate aminotransferase were determined by Synchron-CX automate (Beckman Coulter ${ }^{\circledR}$ ). GH and IGF-1 were measured using immunoradiometric assays (DiaSorin $®$ ). Testosterone and oestradiol were measured by electrochemiluminescence (Roche Diagnostics ${ }^{\circledR}$ ). Specific ELISA kits were used to measure serum levels of leptin (active human leptin, DSL Systems), adiponectin (Quantikine human adiponectin, R\&D Systems), resistin (Quantikine human resistin, R\&D Systems) and ghrelin (human unacylated ghrelin enzyme immunoassay kit, Biovendor).

Body composition was assessed by dual-energy X-ray absorptiometry (Lunar iDXA, GE Healthcare): body fat mass (BFM), body lean mass and fat-free mass compartments were considered. Resting energy expenditure was measured by indirect calorimetry (Quark-RMR-Cosmed ${ }^{\circledR}$ ) in the morning after an overnight fast. Total, resting and active energy expenditure were evaluated using a wearable multisensor armband (SenseWear $^{\circledR}$ Pro2 Armband SWA, BodyMedia, Inc.) ${ }^{(11)}$.

\section{Statistical analysis}

Statistical analysis was carried out using SPSS software (Statistical Package for the Social Sciences, Inc., version 15.0). The results are presented as mean values with their standard errors. The paired Wilcoxon test and the Mann-Whitney $U$ test were used for comparisons between groups. Spearman's correlation was used to test the association among anthropometric, biochemical, hormonal, body composition and energy metabolism parameters in AN patients at T0, T1 and T2. Before the multivariate analyses, variables with skewed distributions were logarithmically transformed to normalise the distributions. Associations were evaluated by linear regression and multiple interactions by stepwise regression and by exhaustive check of all possible multivariate models. The structure of the covariance matrix for each variable was taken into account in all analyses to ensure the most adequate statistical fit and power. A $P<0.05$ was considered statistically significant.

\section{Results}

Comparison between control women and anorexia nervosa women patients at admission (TO)

The mean ages of the AN patients and control subjects were similar at admission. As expected, BMI was significantly lower in the AN group $(P<0 \cdot 05)$ (Table 1$)$. 
Table 1. Anthropometric, biochemical, hormonal, body composition and energy metabolism parameters of control (C) women and patients with anorexia nervosa (AN) at admission and during refeeding

(Mean values with their standard errors)

\begin{tabular}{|c|c|c|c|c|c|c|c|c|}
\hline & \multicolumn{2}{|c|}{$C(n 10)$} & \multicolumn{2}{|c|}{ AN-T0 $(n 11)$} & \multicolumn{2}{|c|}{ AN-T1 $(n 11)$} & \multicolumn{2}{|c|}{ AN-T2 $(n 11)$} \\
\hline & Mean & SEM & Mean & SEM & Mean & SEM & Mean & SEM \\
\hline Age (years) & $24 \cdot 6$ & 0.17 & 21.9 & 0.36 & & & & \\
\hline BMI $\left(\mathrm{kg} / \mathrm{m}^{2}\right)$ & $22 \cdot 3$ & 0.16 & $13 \cdot 1^{*}$ & 0.07 & $16 \cdot 1 \dagger$ & 0.02 & $17 \cdot 8 \ddagger$ & 0.02 \\
\hline ASAT (U/I) & $15 \cdot 4$ & 0.42 & $17 \cdot 2$ & 0.53 & $21 \cdot 1$ & 0.34 & 24.2 & 0.24 \\
\hline ALAT (U/I) & $22 \cdot 5$ & 0.39 & $28 \cdot 1$ & 0.51 & $26 \cdot 3$ & 0.25 & $27 \cdot 8$ & 0.23 \\
\hline Glucose $(\mathrm{mmol} / \mathrm{l})$ & 4.5 & 0.04 & $3.9^{*}$ & 0.05 & $4.5 \dagger$ & 0.03 & $4.9 \ddagger$ & 0.04 \\
\hline Insulin (mIU/l) & $7 \cdot 7$ & 0.49 & $3.0^{*}$ & 0.24 & $7.3 \dagger$ & 0.40 & 9.5 & 0.36 \\
\hline HOMA-IR & 1.5 & 0.06 & $0.5^{\star}$ & 0.05 & $1.4 \dagger$ & 0.09 & $2 \cdot 1 \ddagger$ & $0 \cdot 10$ \\
\hline $\mathrm{GH}(\mathrm{mlU} / \mathrm{l})$ & 8.7 & 0.65 & $24 \cdot 5^{\star}$ & 0.64 & $1.64 t$ & 0.15 & 1.3 & $0 \cdot 11$ \\
\hline IGF-1 (nmol/I & $26 \cdot 3$ & 0.46 & $13 \cdot 7^{*}$ & 0.75 & $27 \cdot 2 \dagger$ & 0.49 & $31.5 \ddagger$ & 0.63 \\
\hline TSH (mlU/l) & 1.5 & 0.10 & $2 \cdot 0$ & $0 \cdot 10$ & $2 \cdot 2$ & 0.08 & $2 \cdot 6$ & 0.08 \\
\hline FT4 (pmol/l) & 13.9 & 0.15 & $12 \cdot 7$ & 0.14 & 11.9 & 0.33 & $12 \cdot 6$ & 0.14 \\
\hline FT3 (pmol/l) & 4.9 & 0.06 & $2 \cdot 4^{*}$ & 0.11 & $5.0 \dagger$ & 0.12 & 4.3 & 0.15 \\
\hline Oestradiol (pmol/l) & 257 & $10 \cdot 50$ & $57 \cdot 1^{*}$ & 4.40 & $154 \dagger$ & $10 \cdot 40$ & 226 & $14 \cdot 30$ \\
\hline Testosterone $(\mathrm{nmol} / \mathrm{l})$ & 1.03 & 0.04 & $1 \cdot 6$ & 0.07 & $0.9 \dagger$ & 0.03 & 0.9 & 0.02 \\
\hline Adiponectin ( $\mu \mathrm{g} / \mathrm{ml})$ & 10.9 & 0.52 & $6 \cdot 7^{*}$ & 0.37 & $17 \cdot 1 \dagger$ & 0.51 & $15 \cdot 1$ & 0.55 \\
\hline Resistin (ng/ml) & 11.8 & 0.38 & $13 \cdot 1$ & 0.80 & 12.5 & 0.48 & $10 \cdot 0$ & 0.32 \\
\hline Leptin (ng/ml) & $11 \cdot 6$ & 0.35 & $0.4^{*}$ & 0.01 & $3 \cdot 1$ & 0.38 & $8.0 \ddagger$ & 0.56 \\
\hline Ghrelin (pg/ml) & 305.0 & 9.60 & $490 \cdot 6^{*}$ & $21 \cdot 2$ & $274.5 \dagger$ & 14.9 & 229.0 & 10.5 \\
\hline BFM (kg) & \multicolumn{2}{|c|}{ ND } & 3.5 & 0.13 & $8.2 \dagger$ & 0.19 & $11 \cdot 8$ & 0.14 \\
\hline BLM $(\mathrm{kg})$ & \multicolumn{2}{|c|}{ ND } & $32 \cdot 3$ & 0.38 & $37 \cdot 2 \dagger$ & 0.29 & 35.9 & 0.27 \\
\hline FFM (kg) & \multicolumn{2}{|c|}{ ND } & 34.1 & 0.37 & 39.3 & 0.25 & 37.9 & 0.30 \\
\hline $\operatorname{REE}(\mathrm{kJ} / \mathrm{d})$ & \multicolumn{2}{|c|}{ ND } & $2840 \cdot 1$ & $66 \cdot 1$ & $4404.9 \dagger$ & $62 \cdot 8$ & $4792.4 \ddagger$ & $70 \cdot 3$ \\
\hline TEE $(\mathrm{kJ} / \mathrm{d})$ & \multicolumn{2}{|c|}{ ND } & 5314.9 & $80 \cdot 3$ & $6364.3 \dagger$ & 71.9 & 6439.6 & 236.9 \\
\hline$A E E(k J / d)$ & \multicolumn{2}{|c|}{ ND } & $419 \cdot 7$ & $40 \cdot 6$ & $865 \cdot 7 \dagger$ & $45 \cdot 2$ & 1603.7 & $96 \cdot 2$ \\
\hline
\end{tabular}

T0, admission (baseline); T1, BMI reached $16 \mathrm{~kg} / \mathrm{m}^{2} ; \mathrm{T} 2$, discharge when BMI reached $17.5 \mathrm{~kg} / \mathrm{m}^{2}$; ASAT, aspartate aminotransferase; ALAT, alanine aminotransferase; HOMA-IR, homeostasis model assessment-insulin resistance; GH, growth hormone; IGF-1, insulin-like growth factor-1; TSH, thyroid-stimulating hormone; FT4, free thyroxine; FT3, free tri-iodothyronine; BFM, body fat mass; ND, not determined; BLM, body lean mass; FFM, fat-free mass; REE, resting energy expenditure; TEE, total energy expenditure; AEE, active energy expenditure.

${ }^{*}$ Mean value was significantly different for $\mathrm{C}$ from that of the AN group before refeeding (AN-T0) $(P<0.05$; Mann-Whitney $U$ test or Wilcoxon test).

† Mean value was significantly different for the first step of refeeding (AN-T1) from that of before refeeding $(\mathrm{AN}-\mathrm{T0})(P<0.05 ; \mathrm{Mann}-$ Whitney $U$ test or Wilcoxon test).

¥Mean value was significantly different for the first step of refeeding (AN-T1) from that of the second step of refeeding (AN-T2) $(P<0.05$; Mann-Whitney $U$ test or Wilcoxon test).

Plasma GH and serum ghrelin levels were higher in AN patients compared with controls $(P<0.05$ for both). Plasma levels of IGF-1, oestradiol, free tri-iodothyronine, leptin and adiponectin were significantly lower in patients with AN compared with control subjects $(P<0.05$ for all). Plasma levels of glucose, insulin and HOMA-IR were significantly lower in patients with AN than in control subjects $(P<0.05$ for all).

\section{Comparison between admission (TO) and first stage of refeeding (T1) in anorexia nervosa patients}

The first stage of refeeding (T1) was reached after a mean of $14 \cdot 1$ (sem 3.3) weeks of hospitalisation (Table 1). As expected, an improvement in body composition was observed after this first period of refeeding. BFM and body lean mass both increased significantly and resting energy expenditure, active energy expenditure and total energy expenditure also increased significantly during this stage $(P<0.05$ for all).

A significant decrease in $\mathrm{GH}$, ghrelin and testosterone levels and an increase in IGF-1, free tri-iodothyronine and oestradiol concentrations were observed $(P<0.05$ for all). Concerning adipocytokine levels, a significant increase was only seen with adiponectin $(P<0.05)$. There was a significant increase in glucose, insulin and HOMA-IR levels $(P<0.05$ for all).

In Spearman's correlation analysis at $\mathrm{T} 1$, there was a significant negative association between $\mathrm{GH}$ and plasma glucose levels and between $\mathrm{GH}$ levels and active energy expenditure $(r-0.7, P=0.04$ and $r-0.8, P=0.02$, respectively).

\section{Comparison between first stage (T1) of refeeding and discharge (T2) in anorexia nervosa patients}

The second stage of refeeding (T2) was reached after a mean hospitalisation of 3.7 (SEM 1.2) weeks from the first stage of refeeding (T1) (Table 1). During this second stage, BFM was the only body composition parameter to increase, but non-significantly. Resting energy expenditure increased significantly $(P<0 \cdot 05)$.

There was a significant increase in IGF-1 and leptin levels $(P<0.05$ for both). Glucose and HOMA-IR levels increased significantly $(P<0.05$ for both).

In Spearman's correlation analysis at T2, plasma GH levels were negatively and significantly associated with leptin levels $(r-0 \cdot 8, P=0 \cdot 03)$. 


\section{Prediction of short-term clinical outcome}

At 6 months after discharge from hospital with a BMI $\geq 17.5 \mathrm{~kg} / \mathrm{m}^{2}$, the AN-R group maintained this objective (BMI $18.08(\operatorname{sem} 0 \cdot 2) \mathrm{kg} / \mathrm{m}^{2}$ ), whereas the AN-NR group did not (BMI $17 \cdot 28(\operatorname{sem} 0 \cdot 2) \mathrm{kg} / \mathrm{m}^{2}$ ). At admission, there were no differences in the total energy intake or in the nutrient intake between the AN-R and the AN-NR groups (2495 (SEM 177) v. 2215 (SEM 134) kJ/d for total energy intake; 30.4 (SEM $2 \cdot 2) v .37 \cdot 0(\operatorname{sem} 4 \cdot 0) \mathrm{g}(20 \cdot 6(\operatorname{sem} 1 \cdot 2) v .25 \cdot 6$ (SEM $2 \cdot 1) \%)$ for protein intake; 73.0 (SEM 7.9) v. 61.2 (SEM 4.9) g (46.6 (SEM 2.5) v. $49.8(\operatorname{sem} 5.0) \%)$ for carbohydrate intake; and 20.8 (SEM 1.2) v. 15.6 (SEM 2.5) g (32.8 (SEM 1.5) v. 24.4 (SEM $3.4) \%$ ) for fat intake). In univariate analysis, the AN-R group differed from the AN-NR group at admission only by $\mathrm{GH}$ levels (39.8 (sem 1.2) v. 5.9 (sem 0.8$) \mathrm{mIU} / 1 ; \quad P<0.05)$ (Fig. 1(a)), but not by IGF-1 levels (14.4 (SEM 1.2) v. 13.9 (sEm 1.5$) \mathrm{nmol} / \mathrm{l}, P=0.65$ ) (Fig. 1(b)) or by the other anthropometric, biochemical, hormonal, body composition and energy metabolism parameters. GH levels were higher in the AN-R group compared with the control group, but not different between the AN-NR and the control groups (Fig. 1(a)).
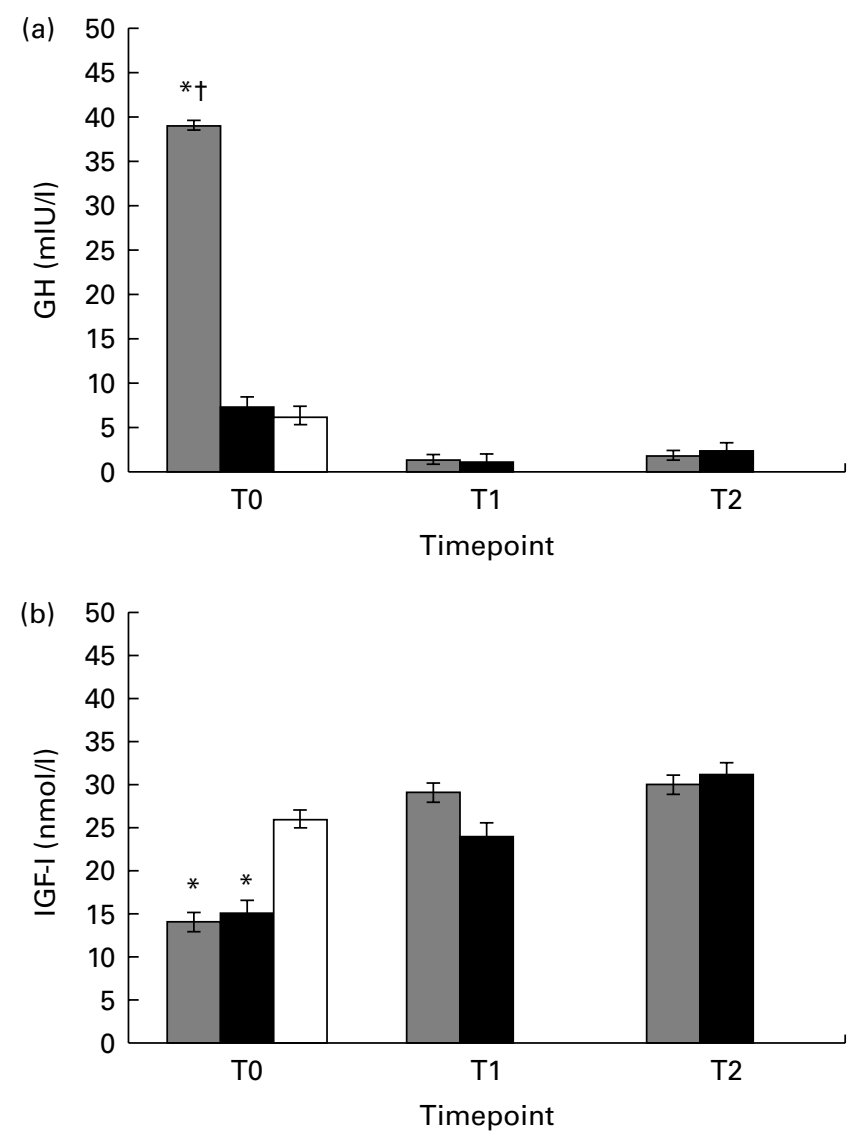

Fig. 1. (a) Plasma levels of growth hormone $(\mathrm{GH})$ and (b) insulin-like growth factor-1 (IGF-1) in control subjects (control, $\square$ ) and in patients with anorexia nervosa in remission (AN-R, $\square$ ) and not in remission (AN-NR, $\square$ ) 6 months after discharge. At admission (T0), at BMI $\geq 16 \mathrm{~kg} / \mathrm{m}^{2}$ (T1) and at $\mathrm{BMI} \geq 17.5$ $\mathrm{kg} / \mathrm{m}^{2}$ (T2). " Mean value was significantly different from that of the control group $(P<0.05$; Mann-Whitney $U$ test). † Mean value was significantly different from that of the AN-NR group $(P<0.05$; Mann-Whitney $U$ test).
IGF-1 levels were lower in the AN-R and AN-NR groups compared with the control group (Fig. 1(b)).

During refeeding, $(\Delta=\mathrm{T} 2-\mathrm{T} 0)$, the AN-R group differed from AN-NR group only by both $\mathrm{GH}$ level decrease $(-15 \cdot 3$ (SEM 2.10) $v$. $-3.6($ SEM 1.84) $\mathrm{mIU} / 1, P<0.05)$ and BMI increase (5.6 (sem 0.3$) v .3 .8($ sem 0.2$\left.) \mathrm{kg} / \mathrm{m}^{2} ; P<0.05\right)$ (Table 2$)$. When we compared the GH level in relation to weight at admission (T0), at T1 and at discharge (T2), we found a higher mean ratio in the AN-R group $v$. the AN-NR group at T0 $(P<0.05)$, but no difference at T1 and T2 between the two groups and a significant decrease of this mean ratio only in the AN-R group between T0 and T1 $(P<0.05)$ and between T0 and T2 $(P<0.05)$. The mean $\Delta \mathrm{GH}(\mathrm{T} 2-\mathrm{T} 0)$ in relation to the 'target weight' at T2 was significantly lower in the AN-R group $v$. the AN-NR group $(P<0.05)$ (Table 3$)$. The difference in BMI increase between the two groups was explained by a non-significant lower BMI in the AN-R group at admission (Table 4) and by a non-significant higher BMI in the AN-R group at discharge.

In an additional stepwise multivariate analysis of BMI at 6-month follow-up (T3), carried out for all the patients and adjusted for $(\Delta=\mathrm{T} 2-\mathrm{T} 0) \Delta$ glucose, $\Delta$ insulin, $\Delta$ HOMA-IR, $\Delta \mathrm{GH}, \Delta \mathrm{IGF}-1, \Delta \mathrm{TSH}, \Delta \mathrm{FT} 4, \Delta$ free tri-iodothyronine, $\Delta$ oestradiol, $\Delta$ testosterone, $\Delta$ adiponectin, $\Delta$ resistin, $\Delta$ leptin, $\Delta$ ghrelin, $\Delta$ BFM, $\Delta$ body lean mass and $\Delta$ fat-free mass, we found that $\Delta \mathrm{GH}$ was associated negatively and significantly with the variability of BMI $(\beta-0.89 ; P<0.05)$ and that $\Delta$ leptin and $\Delta \mathrm{BFM}$ were associated positively and significantly with the variability of BMI $(\beta 0.11 ; P<0.05$ and $\beta 0.39 ; P<0.05$, respectively).

Table 2. Anthropometric, biochemical, hormonal and body composition changes during refeeding (between admission (T0) and discharge (T2)) in anorexia nervosa (AN) patients in remission (AN-R) and not in remission (AN-NR) 6 months after discharge

(Mean values with their standard errors)

\begin{tabular}{|c|c|c|c|c|}
\hline \multirow[b]{2}{*}{$\Delta \mathrm{T} 2-\mathrm{T} 0 \dagger$} & \multicolumn{2}{|c|}{$\mathrm{AN}-\mathrm{R}(n 6)$} & \multicolumn{2}{|c|}{ AN-NR $(n 5)$} \\
\hline & Mean & SEM & Mean & SEM \\
\hline$\Delta \mathrm{BMI}\left(\mathrm{kg} / \mathrm{m}^{2}\right)$ & $5 \cdot 6$ & 0.30 & $3 \cdot 8^{*}$ & 0.20 \\
\hline$\Delta$ Glucose $(\mathrm{mmol} / \mathrm{l})$ & 1.0 & 0.03 & 1.2 & 0.02 \\
\hline$\Delta$ Insulin (mIU/l) & $6 \cdot 1$ & 0.08 & $6 \cdot 2$ & 0.07 \\
\hline$\Delta$ HOMA-IR & 1.5 & 0.03 & 1.6 & 0.07 \\
\hline$\Delta \mathrm{GH}(\mathrm{mlU} / \mathrm{l})$ & $-15 \cdot 3$ & $2 \cdot 10$ & $-3 \cdot 6^{\star}$ & 1.84 \\
\hline$\Delta \mathrm{IGF}-1(\mathrm{nmol} / \mathrm{l})$ & $9 \cdot 2$ & 0.88 & $17 \cdot 2$ & 0.94 \\
\hline$\Delta \mathrm{TSH}(\mathrm{mlU} / \mathrm{l})$ & 0.8 & 0.08 & 0.9 & 0.06 \\
\hline$\Delta \mathrm{FT} 4(\mathrm{pmol} / \mathrm{l})$ & 1.6 & 0.23 & 1.4 & 0.66 \\
\hline$\Delta \mathrm{FT} 3(\mathrm{pmol} / \mathrm{l})$ & $2 \cdot 9$ & 0.08 & $2 \cdot 3$ & 0.09 \\
\hline$\Delta$ Oestradiol (pmol/l) & 141.6 & $10 \cdot 60$ & $339 \cdot 1$ & $26 \cdot 73$ \\
\hline$\Delta$ Testosterone $(\mathrm{nmol} / \mathrm{l})$ & $-1 \cdot 1$ & 0.08 & -0.5 & 0.06 \\
\hline$\Delta$ Adiponectin $(\mu \mathrm{g} / \mathrm{ml})$ & $9 \cdot 7$ & 0.71 & $8 \cdot 3$ & 0.62 \\
\hline$\Delta$ Resistin (ng/ml) & -8.0 & 1.15 & -3.6 & 0.62 \\
\hline$\Delta$ Leptin $(\mathrm{ng} / \mathrm{ml})$ & 11 & 0.93 & 4.9 & 1.09 \\
\hline$\Delta$ Ghrelin (pg/ml) & $-92 \cdot 6$ & $12 \cdot 5$ & -390 & 23.5 \\
\hline$\triangle \mathrm{BFM}(\mathrm{kg})$ & $10 \cdot 1$ & 0.43 & $6 \cdot 2$ & 0.79 \\
\hline$\Delta \mathrm{BLM}(\mathrm{kg})$ & 4.9 & 1.03 & $2 \cdot 8$ & 0.41 \\
\hline$\Delta \mathrm{FFM}(\mathrm{kg})$ & 5.7 & 0.55 & 3.4 & 0.50 \\
\hline
\end{tabular}

HOMA-IR, homeostasis model assessment-insulin resistance; GH, growth hormone; IGF-1, insulin-like growth factor-1; TSH, thyroid-stimulating hormone; FT4, free thyroxine; FT3, free tri-iodothyronine; BFM, body fat mass; BLM, body lean mass; FFM, fat-free mass.

* Mean value was significantly different for AN-R from that of the AN-NR group $(P<0.05$; Mann-Whitney $U$ test $)$.

†T2 - T0 is between admission (T0) and discharge (T2). 
Table 3. Growth hormone $(\mathrm{GH})$ level in relation to weight $(\mathrm{W})$ at admission (T0), at BMl reaching $16 \mathrm{~kg} / \mathrm{m}^{2}$ (T1) and at discharge (T2) of anorexia nervosa (AN) patients in remission (AN-R) and not in remission (AN-NR)

(Individual values and mean values with their standard errors)

\begin{tabular}{|c|c|c|c|c|}
\hline & $\begin{array}{c}\mathrm{GH} / \mathrm{W} \text { at TO } \\
(\mathrm{mlU} / \mathrm{l} \\
\text { per } \mathrm{kg})\end{array}$ & $\begin{array}{c}\mathrm{GH} / \mathrm{W} \text { at } \mathrm{T} 1 \\
(\mathrm{mlU} / \mathrm{l} \\
\text { per } \mathrm{kg})\end{array}$ & $\begin{array}{c}\mathrm{GH} / \mathrm{W} \text { at T2 } \\
(\mathrm{mlU} / \mathrm{l} \\
\text { per } \mathrm{kg})\end{array}$ & $\begin{array}{c}\Delta \mathrm{GH} \\
(\mathrm{T} 2-\mathrm{T} 0) / \mathrm{W} \\
(\mathrm{T} 2)(\%) \S\end{array}$ \\
\hline \multicolumn{5}{|c|}{ AN-R $(n 6)$} \\
\hline 1 & 0.60 & $0 \cdot 12$ & 0.27 & $-33 \cdot 2$ \\
\hline 2 & 0.77 & 0.08 & 0.04 & -73.5 \\
\hline 3 & 0.84 & 0.43 & 0.36 & -48.4 \\
\hline 4 & 1.08 & 0.12 & $0 \cdot 10$ & $-98 \cdot 3$ \\
\hline 5 & $1 \cdot 11$ & 0.35 & 0.39 & $-72 \cdot 1$ \\
\hline 6 & 1.29 & $0 \cdot 10$ & 0.09 & -119.2 \\
\hline Mean & $0.95^{\star}$ & $0.18 \dagger$ & $0.20 \ddagger$ & $-74 \cdot 1^{*}$ \\
\hline SEM & 0.04 & 0.01 & 0.01 & $5 \cdot 4$ \\
\hline \multicolumn{5}{|c|}{ AN-NR $(n 5)$} \\
\hline 1 & 0.21 & $0 \cdot 17$ & 0.15 & $-6 \cdot 1$ \\
\hline 2 & 0.27 & 0.14 & 0.13 & -14.3 \\
\hline 3 & 0.61 & 0.27 & 0.24 & $-35 \cdot 1$ \\
\hline 4 & 0.19 & $0 \cdot 13$ & $0 \cdot 17$ & $-5 \cdot 1$ \\
\hline 5 & $0 \cdot 16$ & $0 \cdot 10$ & 0.14 & $-2 \cdot 1$ \\
\hline Mean & 0.28 & $0 \cdot 16$ & 0.17 & $-11 \cdot 2$ \\
\hline SEM & 0.03 & 0.01 & 0.01 & $2 \cdot 0$ \\
\hline
\end{tabular}

* Mean value was significantly different for AN-R from that of the AN-NR group $(P<0.05$; Mann-Whitney $U$ test or Wilcoxon test).

$\dagger$ Mean value was significantly different for the first step of refeeding (T1) from that of before refeeding (T0) in the AN-R group $(P<0.05$; Mann-Whitney $U$ test or Wilcoxon test).

¥ Mean value was significantly different for the second step of refeeding (T2) from that of before refeeding (TO) in the AN-R group $(P<0.05$; Mann-Whitney $U$ test or Wilcoxon test).

$\S \Delta \mathrm{GH}(\mathrm{T} 2-\mathrm{T} 0)$ is between admission (T0) and discharge (T2).

These three parameters explained $88 \%$ of the variance of BMI at T3 $\left(r^{2} 0 \cdot 88 ; P<0 \cdot 05\right)$, but $\Delta \mathrm{GH}$ was the strongest determinant of BMI at T3, accounting for $74 \%$ of its variability $\left(r^{2} 0 \cdot 74 ; \beta\right.$ $-0 \cdot 71 ; P<0.05)$.

\section{Discussion}

The main original finding of the present study is that GH level at admission and GH evolution during refeeding may predict short-term clinical outcome after weight recuperation. Specifically, low GH levels at admission in hospital and lower amplitude of plasma GH variation between admission and discharge from hospital were strongly associated with relapse 6 months after discharge. In addition, $\mathrm{GH}$ at admission is an important contributor in the variability of the final BMI. It is worthy to note that, despite the small number of patients, clear-cut differences between remitters and non-remitters were observed in this particularly homogeneous population characterised by the recent-onset pure restrictive form of $\mathrm{AN}$ and submitted to a standardised inpatient treatment. This finding should be confirmed by larger and long-term outcome studies. The prognostic effect of $\mathrm{GH}$ variations occurring during refeeding was independent from concomitant IGF-1 changes associated with weight gain. Likewise, whereas higher urinary GH levels have been shown in weight-recovered eumenorrhoeic compared with underweight amenorrhoeic and weight-recovered amenorrhoeic patients, yet, serum IGF-1 levels of amenorrhoeic AN patients were significantly higher only in weight-recovered but still amenorrhoeic patients compared with the underweight amenorrhoeic AN patients ${ }^{(12)}$ The follow-up of the present study was too short to assess the resumption of menses. Moreover, the impossibility to determine 'the healthy weight' corresponding to the weight at the loss of menstruation could represent a limitation of the study that might have an impact on its interpretation. We did not observe any link between the GH level at admission or its evolution during refeeding and any nutritional behaviour before admission or psychiatric co-morbidities. High-protein meals as well as amino acids, such as arginine, lysine, ornithine, tyrosine, glycine and tryptophan, enhance $\mathrm{GH}$ release in human subjects ${ }^{(13)}$, and protein and energy intakes are both essential to regulate IGF- ${ }^{(14)}$. The higher GH level in the AN-R group compared with the AN-NR group at admission cannot be explained by the nutrients, because we found no difference in energy and protein intakes between the two groups. The patients did not receive any medication susceptible to interference with the GH-IGF-1 axis.

The heterogeneity in GH secretion pattern in patients suffering from AN had already been noted by Argente et al. ${ }^{(6)}$, and these authors distinguished two groups characterised by nycthemeral GH values above or below the threshold of $3 \mathrm{ng} / \mathrm{ml}$ for $24 \mathrm{~h}$. However, they were unable to detect any clinical or psychological feature associated with these phenotypes. The functional coupling of GH and IGF-1 has been extensively studied in $\mathrm{AN}^{(15,16)}$. The usual pattern associating low levels of IGF-1, despite an increase of GH secretion, suggests that $\mathrm{AN}$ is characterised by a specific resistance state to the action of GH. Administration of supraphysiological recombinant human $\mathrm{GH}$ treatment to women with AN does not overcome this resistance state to $\mathrm{GH}^{(17)}$. This resistance phenomenon is specific to the GH-IGF-1 axis, as the effect of recombinant human $\mathrm{GH}$ on adipose tissue lipolysis is preserved as the BFM of the treated patients decreased ${ }^{(17)}$. Other authors explained the enhanced GH secretion in AN as a result of an increase in both frequency and amplitude of secretory pulses ${ }^{(18)}$. These anomalies are totally reversed after nutritional recovery ${ }^{(6)}$. Brick et $a l^{(19)}$ have shown that free testosterone could modulate GH secretion across the weight spectrum: AN, normal weight and obesity.

The mechanism of this peripheral resistance to GH, mainly at the liver level, is still unknown. Some explanations based on indirect arguments have been proposed. The decrease in

Table 4. Individual weight and BMI at admission (admission (baseline) (TO)) of anorexia nervosa (AN) patients in remission (AN-R) and not in remission (AN-NR)

(Individual values and mean values with their standard errors)

\begin{tabular}{|c|c|c|c|c|}
\hline & \multicolumn{2}{|c|}{$\mathrm{AN}-\mathrm{R}(n 6)$} & \multicolumn{2}{|c|}{ AN-NR $(n 5)$} \\
\hline & Weight (kg) & $\mathrm{BMI}\left(\mathrm{kg} / \mathrm{m}^{2}\right)$ & Weight (kg) & $\operatorname{BMI}\left(\mathrm{kg} / \mathrm{m}^{2}\right)$ \\
\hline & 34.6 & 11.4 & $37 \cdot 2$ & $13 \cdot 6$ \\
\hline & 36.5 & 13.7 & 39.4 & 14.5 \\
\hline & $42 \cdot 3$ & 13.9 & 31.0 & $12 \cdot 6$ \\
\hline & $36 \cdot 2$ & 13.5 & $33 \cdot 3$ & $11 \cdot 8$ \\
\hline & 33.5 & $12 \cdot 2$ & 38.0 & $14 \cdot 1$ \\
\hline & 34.6 & $13 \cdot 2$ & & \\
\hline Mean & $36 \cdot 1$ & $13 \cdot 0$ & 35.7 & $13 \cdot 3$ \\
\hline SEM & $2 \cdot 1$ & 0.2 & 2.9 & 0.1 \\
\hline
\end{tabular}


the circulating cleaved form of the GH receptor, GH-binding protein, in malnourished patients suffering from AN suggests that expression of $\mathrm{GH}$ receptor could be decreased in the target tissues and that this effect is reversible with refeeding ${ }^{(6,16,20,21)}$. Another hypothesis has recently been proposed attributing a role to the fibroblast growth factor-21 (FGF-21) secreted by the liver ${ }^{(22)}$. Indeed, FGF-21 transgenic mice are smaller than wild-type littermates and are characterised by a profound decrease of IGF-1 without a parallel decrease of GH secretion ${ }^{(23)}$. As FGF-21 is a hormone secreted during prolonged starvation in both animals and human subjects, this factor could explain the $\mathrm{GH}$ resistance associated with $\mathrm{AN}^{(24)}$. Two studies measuring FGF-21 in this disease lead to conflicting results. Dostalova et al. ${ }^{(25)}$ observed a decrease of this factor in patients suffering from AN, with a subsequent reduction after 2 months of refeeding; however, it is worthy to note that they found a positive correlation between the pre-treatment level of FGF-21 and weight gain during refeeding. Fazeli et $a l^{(22)}$ observed similar FGF-21 levels in AN patients and controls before adjusting for insulin resistance and BFM; however, after adjustment for these factors, which could influence FGF-21 production, patients suffering from AN were characterised by a relative increase in FGF-21 levels. Moreover, in the Fazeli et al. study, FGF-21 levels were significantly and positively correlated with $\mathrm{GH}$ AUC and negatively with IGF-1 values, suggesting that, as in rodents, FGF-21 could be a regulator of GH sensitivity in patients with $\mathrm{AN}^{(22)}$. FGF-21 appears to be an important coordinator of the metabolic and hormonal response to prolonged starvation $^{(24)}$. As IGF-1 and FGF-21 are both primarily produced in the liver, we looked for any emaciation-induced liver dysfunctions in the patients characterised by both low levels of GH at admission and poor prognosis. This hypothesis can be ruled out, as aminotransferase (alanine aminotransferase and aspartate aminotransferase) levels at admission were not different between the patients with or without remission. We could assume that the increase of GH associated with active AN could be a marker of the persistence of a correct FGF-21-mediated adapted response to the disease in some patients. Refeeding by shutting down the FGF-21 pathway in these specific patients could lead to a larger weight gain, as shown by Dostalova et al. ${ }^{(25)}$, and to a better prognosis. In contrast, paradoxically low levels of GH in acute AN could be a signal of loss of physiological adaptation associated with poor prognosis.

In conclusion, monitoring GH levels at admission and during refeeding in patients suffering from AN has some prognostic value. The role of this factor in the disease is probably indirect, reflecting specific changes associated with the adapted response to starvation. Elucidating the pathway of this response could be useful in improving the care of patients with AN.

\section{Acknowledgements}

The present research received no specific grant from any funding agency in the public, commercial or not-for-profit sectors. The authors declare no conflict of interest. The authors' responsibilities were as follows: J. P. N., R. V. and B. V. conceived and designed the research, acquired the data, analysed and interpreted the results and drafted the manuscript. A. M. L., C. S.-M., A. B.-L. C, A. N. and H. P. acquired the data, and analysed and interpreted the results. M. M. analysed and interpreted the results. J. G. performed statistical analysis. All the authors read and approved the final manuscript.

\section{References}

1. De Alvaro MT, Munoz-Calvo MT, Barrios V, et al. (2007) Regional fat distribution in adolescents with anorexia nervosa: effect of duration of malnutrition and weight recovery. Eur J Endocrinol 157, 473-479.

2. Haas VK, Kohn MR, Clarke SD, et al. (2009) Body composition changes in female adolescents with anorexia nervosa. Am J Clin Nutr 89, 1005-1010.

3. Lucas AR, Beard CM, O'Fallon WM, et al. (1991) 50-year trends in the incidence of anorexia nervosa in Rochester, Minn.: a population-based study. Am J Psychiatry 148, 917-922.

4. Misra M \& Klibanski A (2010) Neuroendocrine consequences of anorexia nervosa in adolescents. Endocr Dev 17, $197-214$.

5. Miller CA \& Golden NH (2010) An introduction to eating disorders: clinical presentation, epidemiology, and prognosis. Nutr Clin Pract 25, 110-115.

6. Argente J, Caballo N, Barrios V, et al. (1997) Multiple endocrine abnormalities of the growth hormone and insulin-like growth factor axis in patients with anorexia nervosa: effect of short- and long-term weight recuperation. J Clin Endocrinol Metab 82, 2084-2092.

7. Warren MP (2011) Endocrine manifestations of eating disorders. J Clin Endocrinol Metab 96, 333-343.

8. Meguerditchian C, Samuelian-Massat C, Valero $\mathrm{R}$, et al. (2009) The impact of weight normalization on quality of recovery in anorexia nervosa. J Am Coll Nutr 28, 397-404.

9. Treasure J, Claudino AM \& Zucker N (2010) Eating disorders. Lancet 375, 583-593.

10. Becker AE, Grinspoon SK, Klibanski A, et al. (1999) Eating disorders. N Engl J Med 340, 1092-1098.

11. Berntsen S, Hageberg R, Aandstad A, et al. (2008) Validity of physical activity monitors in adults participating in free living activities. Br J Sports Med 44, 657-664.

12. Audi L, Mantzoros CS, Vidal-Puig A, et al. (1998) Leptin in relation to resumption of menses in women with anorexia nervosa. Mol Psychiatry 3, 544-547.

13. Scacchi M, Ida Pincelli A \& Cavagnini F (2003) Nutritional status in the neuroendocrine control of growth hormone secretion: the model of anorexia nervosa. Front Neuroendocrinol 24, 200-224.

14. Thissen JP, Underwood LE \& Ketelslegers JM (2003) Regulation of insulin-like growth factor-1 in starvation and injury. Nutr Rev 57, 167-176.

15. Golden NH, Kreitzer P, Jacobson MS, et al. (1994) Disturbances in growth hormone secretion and action in adolescents with anorexia nervosa. $J$ Pediatr 125, 655-660.

16. Misra M, Miller KK, Bjornson J, et al. (2003) Alterations in growth hormone secretory dynamics in adolescent girls with anorexia nervosa and effects on bone metabolism. J Clin Endocrinol Metab 88, 5615-5623.

17. Fazeli PK, Lawson EA, Prabhakaran R, et al. (2010) Effects of recombinant human growth hormone in anorexia nervosa: a randomized, placebo-controlled study. J Clin Endocrinol Metab 95, 4889-4897. 
18. Scacchi M, Pincelli AI, Caumo A, et al. (1997) Spontaneous nocturnal growth hormone secretion in anorexia nervosa. J Clin Endocrinol Metab 82, 3225-3229.

19. Brick DJ, Gerweck AV, Meenaghan E, et al. (2010) Determinants of IGF1 and GH across the weight spectrum: from anorexia nervosa to obesity. Eur J Endocrinol 163, 185-191.

20. Counts DR, Gwirtsman H, Carlsson LM, et al. (1992) The effect of anorexia nervosa and refeeding on growth hormone-binding protein, the insulin-like growth factors (IGFs), and the IGF-binding proteins. J Clin Endocrinol Metab 75, 762-767.

21. Llopis MA, Granada ML, Cuatrecasas G, et al. (1998) Growth hormone-binding protein directly depends on serum leptin levels in adults with different nutritional status. J Clin Endocrinol Metab 83, 2006-2011.

22. Fazeli PK, Misra M, Goldstein M, et al. (2010) Fibroblast growth factor-21 may mediate growth hormone resistance in anorexia nervosa. J Clin Endocrinol Metab 95, 369-374.

23. Inagaki T, Lin VY, Goetz R, et al. (2008) Inhibition of growth hormone signaling by the fasting-induced hormone FGF21. Cell Metab 8, 77-83.

24. Kliewer SA \& Mangelsdorf DJ (2010) Fibroblast growth factor 21: from pharmacology to physiology. Am J Clin Nutr 91 , 254S-257S.

25. Dostalova I, Kavalkova P, Haluzikova D, et al. (2008) Plasma concentrations of fibroblast growth factors 19 and 21 in patients with anorexia nervosa. J Clin Endocrinol Metab 93, 3627-3632. 